Vietnam Journal of Mechanics, VAST, Vol.40, No. 3 (2018), pp. 265-283

DOI: https://doi.org/10.15625/0866-7136/11366

\title{
OPTIMAL CONTROL OF TRANSVERSE VIBRATION OF EULER-BERNOULLI BEAM WITH MULTIPLE DYNAMIC VIBRATION ABSORBERS USING TAGUCHI'S METHOD
}

\author{
Nguyen Van Khang ${ }^{1, *}$, Vu Duc Phuc ${ }^{2}$, Nguyen Thi Van Huong ${ }^{1}$, Do The Duong ${ }^{1}$ \\ ${ }^{1}$ Hanoi University of Science and Technology, Vietnam \\ ${ }^{2}$ Hung Yen University of Technology and Education, Vietnam \\ *E-mail: khang.nguyenvan2@hust.edu.vn \\ Received February 22, 2018
}

\begin{abstract}
Vibration absorbers are frequently used to suppress the excessive vibrations in structural systems. In this paper, an imposing nodes technique is applied for vibration suppression of Euler-Bernoulli beams subjected to forced harmonic excitations by means of multiple dynamic vibration absorbers. A procedure based on Taguchi's method is proposed to determine the optimum absorber parameters to suppress the vibration amplitude of the beams. Numerical tests are performed to show the effectiveness of the proposed procedure.
\end{abstract}

Keywords: beam structures, dynamic vibration absorber, Taguchi's method, harmonic excitations, passive vibration control.

\section{INTRODUCTION}

Beams are conventional constructions such as house beams, suspended cable in suspension structures, air traffic control towers, wind turbine columns, etc. In the course of the work, these structures are exposed to the effects of wind exploitation and operation. Under the influence of changing external frequencies, beam structures appear to be subjected to forced vibration. Since the frequency of the external force changes over a wide band, there is the possibility of a resonance which can cause structural damage. Therefore, the reduction of the amplitude range of the structure at resonant frequency is a necessary task. In order to determine the resonance frequencies and to investigate the behavior of the system under the action of external forces, the natural frequencies of the structure must first be evaluated [1,2]. Vibration amplitudes at different points in the structure excited by the external force in a wide frequency band are then calculated. Dynamic dampers are usually used to reduce the vibrations of the beams.

Besides, undesirable vibrations in mechanical structures have been effectively reduced by the application of dynamic vibration absorbers (DVA). The reasons for those

(C) 2018 Vietnam Academy of Science and Technology 
applications of the DVA are its efficient, reliable and low-cost characteristics $[3,4]$. Recently, considerable effort has been devoted to devise effective control strategies for this task. Jacquot [5] developed a technique to give the optimal parameters of DVA for the elimination of excessive vibration in sinusoidally forced Bernoulli-Euler beams. Ozguven and Candir [6] presented a procedure for determining the optimum parameters of two DVAs attached to a beam to suppress any two resonances. Lin and Cho [7] investigated dynamic characteristics of a simply supported beam traversed by multiple moving loads and a practical scheme for suppressing the resulting resonant or excessive vibration by using a damped absorber. In [8] Chtiba and his colleagues have proposed a new strategy for the optimal design of supplementary absorbers that warrant confinement with and without suppression of vibration in flexible structures. In [9] Vestroni et al. studied the pedestrian-induced vibrations in suspension footbridges via multiple tuned mas dampers. The solutions to $H_{\infty}$ and $H_{2}$ optimization problems of a variant dynamic absorber applied to suppress vibration in beam structures are derived analytically by

Noori and Farshidianfar [10]. The calculating results of Samani and his colleagues [11] show that, for the test cases considered, the DVAs with essentially nonlinear stiffnesses having higher power are more effective than the linear one in reducing the maximum beam deflection. In [12] Patil and Awasare have used variable stiffness vibration neutralizers to impose zero displacement or nodes to reduce vibration at desired locations on a Euler-Bernoulli beam subjected to forced harmonic excitation. Latas $[13,14]$ discussed the problem of optimal choice of position and parameters of the system of translational and rotational dynamic absorbers in beams.

The study of optimal design of parameters of dynamic vibration absorber installed in beam structures becomes an interesting problem in recent years. It is well known that Taguchi's method for the product design process may be divided into three stages: system design, parameter design, and tolerance design [15-22]. Taguchi's method of parameter design is successfully applied to many mechanical systems: acoustic muffler, gear/pinion system, spring, electro-hydraulic servo system, dynamic vibration absorber. In each system, the design parameters to be optimized are identified, along with the desired response. The present study deals with the determination of the optimal parameters of DVAs for the vibration reduction of Euler-Bernoulli beams subjected to forced harmonic excitation using Taguchi's method. The target function is determined by suppressing the resonance vibrations of beams.

\section{DERIVATION OF TRANSVERSE VIBRATION EQUATIONS OF BEAM WITH DYNAMIC VIBRATION ABSORBERS}

Let us consider the model of a Euler-Bernoulli beam of the length $\mathrm{L}$ and the flexural rigidity EI, which is attached with a number of DVAs at positions $x=\eta_{j}\left(j=1, \ldots, n_{a}\right)$ as shown in Fig. 1. For simplicity, it is assumed that the considered beam is homogeneous with a uniform cross section, where $w$ is the dynamic deflection, $u_{j}$ is vertical coordinate of $j$-absorber, $k$ are stiffness, damping coefficients and mass of $j$-absorber, respectively, and $p(x, t)$ is the distributed force.

Using the method of substructures, the system is now divided into $n_{a}+1$ substructures, namely, the beam structure and $n_{a}$ absorbers (Fig. 2). 


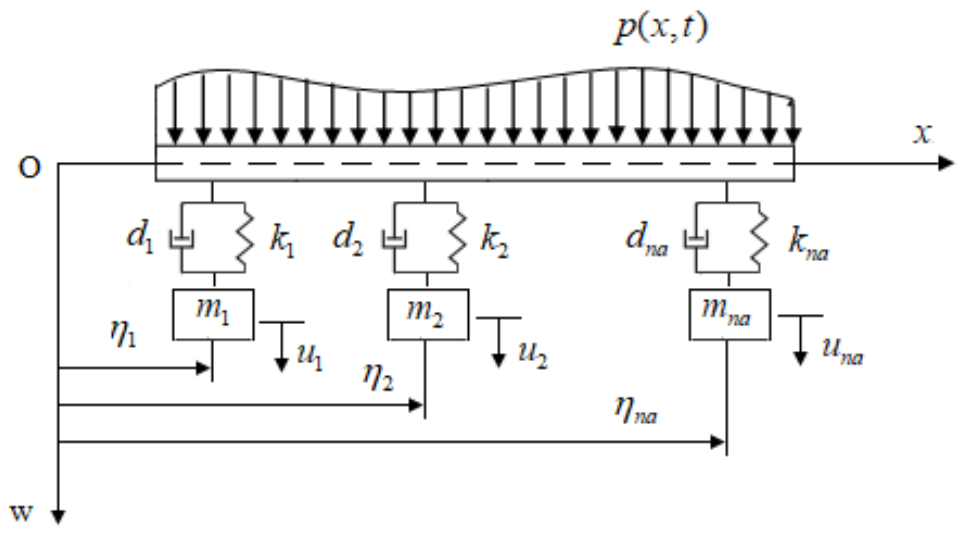

Fig. 1. Beam with dynamic vibration absorbers
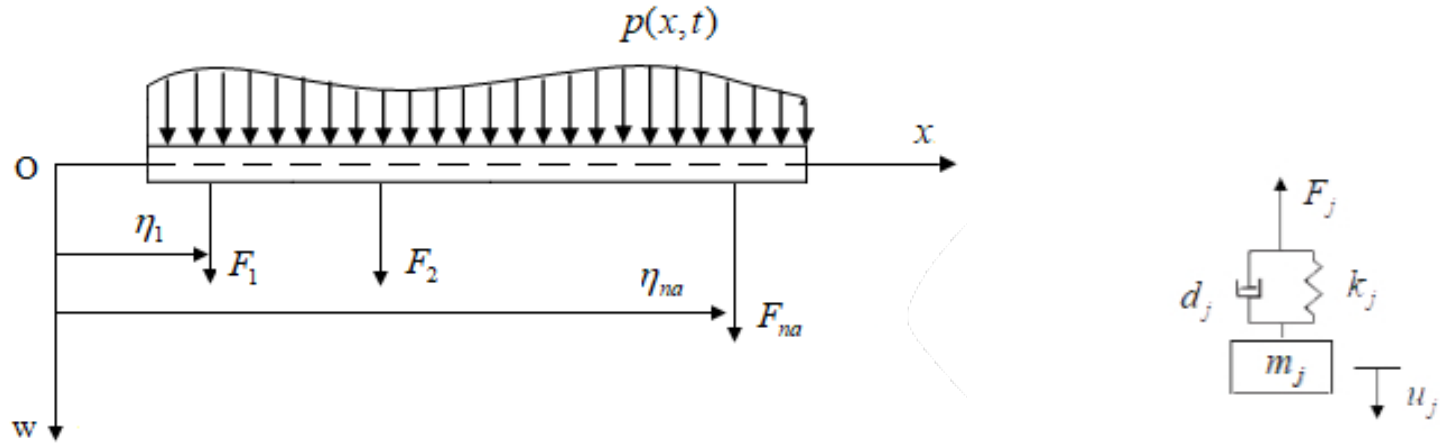
(a) Beam
(b) Absorber

Fig. 2. Substructures

Reaction forces have the following form

$$
F_{j}(t)=k_{j}\left(u_{j}-w_{\eta j}\right)+d_{j}\left(\dot{u}_{j}-\dot{w}_{\eta j}\right), \quad j=\left(1,2, \ldots, n_{a}\right),
$$

where

$$
w_{\eta j}=w\left(\eta_{j}, t\right), \quad \dot{w}_{\eta j}=\frac{\partial w\left(\eta_{j}, t\right)}{\partial t} .
$$

Using Newton's second law, the equation describing the vibration of $j$-absorber can be expressed in the form

$$
m_{j} \ddot{u}_{j}=-F_{j} \Rightarrow F_{j}(t)=-m_{j} \ddot{u}_{j} .
$$

Substitution of Eq. (2) into Eq. (1) yields the vibration equation of $j$-absorber

$$
m_{j} \ddot{u}_{j}+d_{j} \dot{u}_{j}+k_{j} u_{j}=k_{j} w_{\eta j}+d_{j} \dot{w}_{\eta j}, \quad\left(j=1,2, \ldots, n_{a}\right) .
$$


Applying the basic principles of dynamics, the equation that describes transverse vibration of beam including internal friction is [1,2]

$$
\mu\left(\frac{\partial^{2} w}{\partial t^{2}}+c^{(e)} \frac{\partial w}{\partial t}\right)+E I\left[\frac{\partial^{4} w}{\partial x^{4}}+c^{(i)} \frac{\partial^{5} w}{\partial x^{4} \partial t}\right]=p(x, t)+\sum_{j=1}^{n_{a}} F_{j} \delta\left(x-\eta_{j}\right) .
$$

In Eq. (4), $\mu$ denotes mass per length unit, $c^{(e)}, c^{(i)}$ are damping coefficient and internal friction coefficient per length unit of beam, respectively, and the Delta-Dirac function $\delta\left(x-\eta_{j}\right)$ is defined by

$$
\delta\left(x-\eta_{j}\right)= \begin{cases}1 & \text { when } x=\eta_{j} \\ 0 & \text { when } x \neq \eta_{j}\end{cases}
$$

The vibration equations according to Eqs. (3) and (4) are a mixed set of ordinary and partial differential equations. Four boundary conditions, two at $x=0$ and two at $x=L$, and the initial conditions must be specified to find the solution of this set. form

Using Ritz-Galerkin method, the solution of Eqs. (3) and (4) can be found in the

$$
w(x, t)=\sum_{r=1}^{n_{b}} X_{r}(x) q_{r}(t),
$$

where $X_{r}(x)$ denotes the mode shape of the beam and $q_{r}(t)$ is the generalized displacement to be determined. Substituting Eq. (6) into Eqs. (4) and (3), we find

$$
\begin{aligned}
\ddot{q}_{k}(t)+ & \left(c^{(e)}+c^{(i)} \omega_{k}^{2}\right) \dot{q}_{k}(t)+\omega_{k}^{2} q_{k}(t) \\
& =\frac{\int_{0}^{l} p(x, t) X_{k}(x) \mathrm{d} x}{\mu \int_{0}^{l} X_{k}^{2}(x) \mathrm{d} x}+\frac{\sum_{j=1}^{n_{a}} \int_{0}^{l} F_{j}(t) X_{k}(x) \delta\left(x-\eta_{j}\right) \mathrm{d} x}{\mu \int_{0}^{l} X_{k}^{2}(x) \mathrm{d} x}, \quad\left(k=1, \ldots, n_{b}\right)
\end{aligned}
$$

and

$$
m_{j} \ddot{u}_{j}(t)+d_{j} \dot{u}_{j}(t)+k_{j} u_{j}(t)-d_{j} \sum_{r=1}^{n_{b}} X_{r}\left(\eta_{j}\right) \dot{q}_{r}(t)-k_{j} \sum_{r=1}^{n_{b}} X_{r}\left(\eta_{j}\right) q_{r}(t)=0, \quad\left(j=1,2, \ldots, n_{a}\right)
$$

where $\omega_{k}$ is eigenfrequency of the beam $[1,2]$. Using the notations

$$
2 \delta_{k}=\left(c^{(e)}+c^{(i)} \omega_{k}^{2}\right), \quad D_{k}=\mu \int_{0}^{l} X_{k}^{2}(x) \mathrm{d} x=\text { const. }
$$

it follows from Eq. (7) that

$$
\ddot{q}_{k}(t)+2 \delta_{k} \dot{q}_{k}(t)+\omega_{k}^{2} q_{k}(t)=h_{k}(t)+\frac{1}{D_{k}} \sum_{j=1}^{n_{a}} \int_{0}^{l} F_{j}(t) X_{k}(x) \delta\left(x-\eta_{j}\right) \mathrm{d} x .
$$




$$
\alpha_{k}(t)=\int_{0}^{l} p(x, t) X_{k}(x) \mathrm{d} x, \quad h_{k}(t)=\frac{\alpha_{k}(t)}{D_{k}}
$$

Substitution of Eq. (6) into Eq. (1) yields

$$
\begin{aligned}
F_{j}(t) & =d_{j}\left[\dot{u}_{j}(t)-\dot{w}_{\eta j}\right]+k_{j}\left[u_{j}(t)-w_{\eta j}\right] \\
& =d_{j} \dot{u}_{j}(t)+k_{j} u_{j}(t)-d_{j} \sum_{r=1}^{n_{b}} X_{r}\left(\eta_{j}\right) \dot{q}_{r}(t)-k_{j} \sum_{r=1}^{n_{b}} X_{r}\left(\eta_{j}\right) q_{r}(t),
\end{aligned}
$$

in which $d_{j}, k_{j}, X_{r}\left(\eta_{j}\right)\left(j=1,2, \ldots, n_{a}\right)$ are the known constants. According to the property of the Delta-Dirac function we have

$$
\sum_{j=1}^{n_{a}} \int_{0}^{l} F_{j}(t) X_{k}(x) \delta\left(x-\eta_{j}\right) \mathrm{d} x=\sum_{j=1}^{n_{a}} F_{j}(t) X_{k}\left(\eta_{j}\right) .
$$

Substitution of Eq. (12) into Eq. (13) one obtains

$$
\begin{aligned}
\sum_{j=1}^{n_{a}} F_{j}(t) X_{k}\left(\eta_{j}\right)= & \sum_{j=1}^{n_{a}} d_{j} \dot{u}_{j}(t) X_{k}\left(\eta_{j}\right)+\sum_{j=1}^{n_{a}} k_{j} u_{j}(t) X_{k}\left(\eta_{j}\right) \\
& -\sum_{j=1}^{n_{a}} \sum_{r=1}^{n_{b}} d_{j} X_{r}\left(\eta_{j}\right) X_{k}\left(\eta_{j}\right) \dot{q}_{r}(t)-\sum_{j=1}^{n_{a}} \sum_{r=1}^{n_{b}} k_{j} X_{r}\left(\eta_{j}\right) X_{k}\left(\eta_{j}\right) q_{r}(t) \\
= & \sum_{j=1}^{n_{a}} X_{k}\left(\eta_{j}\right)\left[d_{j} \dot{u}_{j}(t)+k_{j} u_{j}(t)\right]-\sum_{j=1}^{n_{a}} X_{k}\left(\eta_{j}\right)\left\{\sum_{r=1}^{n_{b}}\left[d_{j} \dot{q}_{r}(t)+k_{j} q_{r}(t)\right] X_{r}\left(\eta_{j}\right)\right\} .
\end{aligned}
$$

Substitution of Eq. (14) into Eq. (10) yields

$$
\begin{aligned}
\ddot{q}_{k}(t) & +2 \delta_{k} \dot{q}_{k}(t)+\omega_{k}^{2} q_{k}(t)=h_{k}(t)+\frac{1}{D_{k}} \sum_{j=1}^{n_{a}} X_{k}\left(\eta_{j}\right)\left[d_{j} \dot{u}_{j}(t)+k_{j} u_{j}(t)\right] \\
& -\frac{1}{D_{k}} \sum_{j=1}^{n_{a}} X_{k}\left(\eta_{j}\right)\left\{\sum_{r=1}^{n_{b}}\left[d_{j} \dot{q}_{r}(t)+k_{j} q_{r}(t)\right] X_{r}\left(\eta_{j}\right)\right\}, \quad\left(k=1,2, \ldots, n_{b}\right) .
\end{aligned}
$$

It follows from Eq. (8) that

$$
\sum_{r=1}^{n_{b}}\left[d_{j} \dot{q}_{r}(t)+k_{j} q_{r}(t)\right] X_{r}\left(\eta_{j}\right)=m_{j} \ddot{u}_{j}(t)+d_{j} \dot{u}_{j}(t)+k_{j} u_{j}(t)
$$

By substituting Eq. (16) into Eq. (15) leads to

$$
\begin{aligned}
\ddot{q}_{k}(t) & +2 \delta_{k} \dot{q}_{k}(t)+\omega_{k}^{2} q_{k}(t)=h_{k}(t)+\frac{1}{D_{k}} \sum_{j=1}^{n_{a}} X_{k}\left(\eta_{j}\right)\left[d_{j} \dot{u}_{j}(t)+k_{j} u_{j}(t)\right] \\
& -\frac{1}{D_{k}} \sum_{j=1}^{n_{a}} X_{k}\left(\eta_{j}\right)\left\{m_{j} \ddot{u}_{j}(t)+d_{j} \dot{u}_{j}(t)+k_{j} u_{j}(t)\right\}, k=1,2, \ldots, n_{b} .
\end{aligned}
$$


Eqs. (17) and (8) consist of a system of $n=n_{a}+n_{b}$ ordinary differential equations that describes the vibration of the beam with dynamic vibration absorbers.

We consider now the vibration of the beams under harmonic excitation $p(t)=p_{0}$ $\sin \Omega t$. According to Eqs. (8) and (11) we have

$$
\alpha_{k}(t)=\left[p_{0} \int_{0}^{l} X_{k}(x) \mathrm{d} x\right] \sin \Omega t, \quad h_{k}(t)=\frac{p_{0} \int_{0}^{l} X_{k}(x) \mathrm{d} x}{D_{k}} \sin \Omega t .
$$

It follows that

$$
h_{k}(t)=\widehat{h}_{k} \sin \Omega t, \quad \widehat{h}_{k}=\frac{p_{0} \int_{0}^{l} X_{k}(x) \mathrm{d} x}{D_{k}} .
$$

In this case, Eq. (17) has the following form

$$
\ddot{q}_{k}(t)+2 \delta_{k} \dot{q}_{k}(t)+\omega_{k}^{2} q_{k}(t)=\widehat{h}_{k} \sin \Omega t-\frac{1}{D_{k}} \sum_{j=1}^{n_{a}} m_{j} X_{k}\left(\eta_{j}\right) \ddot{u}_{j}, \quad k=\left(1,2 \ldots, n_{b}\right) .
$$

Using the notations

it follows from Eq. (8) that

$$
\frac{d_{j}}{m_{j}}=2 \delta_{j c}, \quad \frac{k_{j}}{m_{j}}=\omega_{j c}^{2}
$$

$\ddot{u}_{j}(t)+2 \delta_{j c} \dot{u}_{j}(t)+\omega_{j c}^{2} u_{j}(t)=2 \delta_{j c} \sum_{r=1}^{n_{b}} X_{r}\left(\eta_{j}\right) \dot{q}_{r}(t)+\omega_{j c}^{2} \sum_{r=1}^{n_{b}} X_{r}\left(\eta_{j}\right) q_{r}(t), \quad\left(j=1,2, \ldots, n_{a}\right)$.

Eqs. (19) and (20) are a system of $n=n_{a}+n_{b}$ differential equations describing the transverse vibration of beam with a lot of dynamic vibration absorbers under the harmonic distributed force, in which $X_{k}(x)$ is the eigenfunction of beam. The concrete form of $X_{k}(x)$ depends on the boundary conditions of beam.

\section{THE COMPLEX FREQUENCY RESPONSE FUNCTION}

In this section, we consider cases that often occur in structural, when the excitation frequency $\Omega$ is approximately equal to the fundamental frequency $\omega_{1}$ of the beam $(\Omega \approx$ $\left.\omega_{1}\right)$. It follows from Eq. (19) and Eq. (20) that

$$
\begin{gathered}
\ddot{q}_{1}(t)+2 \delta_{1} \dot{q}_{1}(t)+\omega_{1}^{2} q_{1}(t)=\widehat{h}_{1} \sin \Omega t-\frac{1}{D_{1}} \sum_{j=1}^{n_{a}} m_{j} X_{k}\left(\eta_{j}\right) \ddot{u}_{j}, \\
\ddot{u}_{j}(t)+2 \delta_{j c} \dot{u}_{j}(t)+\omega_{j c}^{2} u_{j}(t)=2 \delta_{j c} X_{1}\left(\eta_{j}\right) \dot{q}_{1}(t)+\omega_{j c}^{2} X_{1}\left(\eta_{j}\right) q_{1}(t), \quad\left(j=1,2, \ldots, n_{a}\right) .
\end{gathered}
$$

We use the following notations

$$
x_{s}=q_{1}, \quad \omega_{s}=\omega_{1}, \quad \delta_{s}=\delta_{1}, \quad \widehat{h}_{s}=\widehat{h}_{1},
$$


Eq. (21) can be written in the following form

$$
\ddot{x}_{s}(t)+2 \delta_{s} \dot{x}_{s}(t)+\omega_{s}^{2} x_{s}(t)=\widehat{h}_{1} \sin \Omega t-\frac{1}{D_{1}} \sum_{j=1}^{n_{a}} m_{j} X_{1}\left(\eta_{j}\right) \ddot{u}_{j} .
$$

The solution of Eqs. (22) and (24) can now be found using the method of frequency response function. We note that $\cos \Omega t=\operatorname{Re} e^{i \Omega t}, \sin \Omega t=\operatorname{Im} e^{i \Omega t}$, Eq. (24) can thus be written as follows

$$
\ddot{x}_{s}(t)+2 \delta_{s} \dot{x}_{s}(t)+\omega_{s}^{2} x_{s}(t)=\widehat{h}_{s} e^{i \Omega t}-\frac{1}{D_{1}} \sum_{j=1}^{n_{a}} m_{j} X_{1}\left(\eta_{j}\right) \ddot{u}_{j} .
$$

We find the solutions of Eqs. (22) and (25) in the form

$$
x_{s}(t)=H_{s} e^{i \Omega t}, u_{j}(t)=H_{j c} e^{i \Omega t} .
$$

Substitution of Eq. (26) into Eqs. (25) and (22) lead to the system of linear algebraic equations

$$
\begin{gathered}
{\left[\omega_{s}^{2}-\Omega^{2}+i 2 \delta_{s} \Omega\right] H_{s}=\widehat{h}_{s}+\frac{\Omega^{2}}{D_{1}} \sum_{j=1}^{n_{a}} m_{j} X_{1}\left(\eta_{j}\right) H_{j c},} \\
{\left[\omega_{j c}^{2}-\Omega^{2}+2 i \delta_{j c} \Omega\right] H_{j c}=\left[\omega_{j c}^{2}+2 i \delta_{j c} \Omega\right] X_{1}\left(\eta_{j}\right) H_{s}, \quad\left(j=1,2, \ldots, n_{a}\right) .}
\end{gathered}
$$

From Eq. (28) one has

$$
H_{j c}=\frac{\left[\omega_{j c}^{2}+i 2 \delta_{j c} \Omega\right]}{\left[\omega_{j c}^{2}-\Omega^{2}+i 2 \delta_{j c} \Omega\right]} X_{1}\left(\eta_{j}\right) H_{s}, \quad\left(j=1,2, \ldots, n_{a}\right) .
$$

Substitution of Eq. (29) into Eq. (27) yields

$$
\left[\omega_{s}^{2}-\Omega^{2}+i 2 \delta_{s} \Omega\right] H_{s}=\widehat{h}_{s}+H_{s} \frac{\Omega^{2}}{D_{1}} \sum_{j=1}^{n_{a}}\left(m_{j} X_{1}^{2}\left(\eta_{j}\right) \frac{\left[\omega_{j c}^{2}+i 2 \delta_{j c} \Omega\right]}{\left[\omega_{j c}^{2}-\Omega^{2}+i 2 \delta_{j c} \Omega\right]}\right) .
$$

By introducing

$$
a_{11}=\sum_{j=1}^{n_{a}} m_{j} X_{1}^{2}\left(\eta_{j}\right) \frac{\left[\omega_{j c}^{2}+i 2 \delta_{j c} \Omega\right]}{\left[\omega_{j c}^{2}-\Omega^{2}+i 2 \delta_{j c} \Omega\right]} .
$$

Eq. (30) can be written in the following form

$$
\left(\left[\omega_{s}^{2}-\Omega^{2}+i 2 \delta_{s} \Omega\right]+\frac{\Omega^{2}}{D_{k}} a_{11}\right) H_{s}=\widehat{h}_{s}
$$

From Eq. (32) one has

$$
H_{s}=\frac{\widehat{h}_{s}}{\left[\omega_{s}^{2}-\Omega^{2}+i 2 \delta_{s} \Omega\right]+\frac{\Omega^{2}}{D_{1}} a_{11}},
$$


in which

$$
\begin{aligned}
a_{11} & =\sum_{j=1}^{n_{a}} m_{j} X_{1}^{2}\left(\eta_{j}\right) \frac{\left[\omega_{j c}^{2}+i 2 \delta_{j c} \Omega\right]}{\left[\omega_{j c}^{2}-\Omega^{2}+i 2 \delta_{j c} \Omega\right]} \\
& =\sum_{j=1}^{n_{a}} m_{j} X_{1}^{2}\left(\eta_{j}\right) \frac{\left[\omega_{j c}^{2}+i 2 \delta_{j c} \Omega\right]\left[\omega_{j c}^{2}-\Omega^{2}-i 2 \delta_{j c} \Omega\right]}{\left[\left(\omega_{j c}^{2}-\Omega^{2}\right)^{2}+4 \delta_{j c}^{2} \Omega^{2}\right]} \\
& =\sum_{j=1}^{n_{a}} m_{j} X_{1}^{2}\left(\eta_{j}\right) \frac{\left[\omega_{j c}^{2}\left(\omega_{j c}^{2}-\Omega^{2}\right)+4 \delta_{j c}^{2} \Omega^{2}-2 i \delta_{j c} \Omega^{3}\right]}{\left[\left(\omega_{j c}^{2}-\Omega^{2}\right)^{2}+4 \delta_{j c}^{2} \Omega^{2}\right]} .
\end{aligned}
$$

The denominator in Eq. (33) takes the form

$$
\begin{aligned}
& {\left[\omega_{s}^{2}-\Omega^{2}+i 2 \delta_{1} \Omega\right]+\frac{\Omega^{2}}{D_{1}} a_{11}=\left[\omega_{s}^{2}-\Omega^{2}+i 2 \delta_{s} \Omega\right]} \\
& +\frac{\Omega^{2}}{D_{1}} \sum_{j=1}^{n_{a}} m_{j} X_{1}^{2}\left(\eta_{j}\right) \frac{\left[\omega_{j c}^{2}\left(\omega_{j c}^{2}-\Omega^{2}\right)+4 \delta_{j c}^{2} \Omega^{2}\right]}{\left[\left(\omega_{j c}^{2}-\Omega^{2}\right)^{2}+4 \delta_{j c}^{2} \Omega^{2}\right]}-i \sum_{j=1}^{n_{a}} m_{j} X_{1}^{2}\left(\eta_{j}\right) \frac{2 \Omega^{3} \delta_{j c}}{\left[\left(\omega_{j c}^{2}-\Omega^{2}\right)^{2}+4 \delta_{j c}^{2} \Omega^{2}\right]} .
\end{aligned}
$$

By introducing the following notations

$$
\begin{gathered}
a=\left[\omega_{s}^{2}-\Omega^{2}\right]+\frac{\Omega^{2}}{D_{1}} \sum_{j=1}^{n_{a}} m_{j} X_{1}^{2}\left(\eta_{j}\right) \frac{\left[\omega_{j c}^{2}\left(\omega_{j c}^{2}-\Omega^{2}\right)+4 \delta_{j c}^{2} \Omega^{2}\right]}{\left[\left(\omega_{j c}^{2}-\Omega^{2}\right)^{2}+4 \delta_{j c}^{2} \Omega^{2}\right]}, \\
b=2 \delta_{s} \Omega-\frac{\Omega^{2}}{D_{1}} \sum_{j=1}^{n_{a}} m_{j} X_{1}^{2}\left(\eta_{j}\right) \frac{2 \Omega^{3} \delta_{j c}}{\left[\left(\omega_{j c}^{2}-\Omega^{2}\right)^{2}+4 \delta_{j c}^{2} \Omega^{2}\right]},
\end{gathered}
$$

function $H_{S}$ can be written in the form

$$
H_{s}=\frac{\widehat{h}_{s}}{a+i b}=\frac{\widehat{h}_{s}(a-i b)}{a^{2}+b^{2}} .
$$

The modulus of the complex frequency response function $H_{S}$ can now be calculated by the following formula

$$
H=\left|\widetilde{H}_{s}\right|=\frac{\widehat{h}_{s}}{\sqrt{a^{2}+b^{2}}} .
$$

The optimum problem is stated as follows: Find the parameters of dynamic vibration absorbers $m_{j}, c_{j}, k_{j}\left(j=1,2, \ldots, n_{a}\right)$ which minimize the objective function according to Eq. (38). 


\section{USING THE TAGUCHI METHOD FOR DETERMINATION OF THE OPTIMUM ABSORBER PARAMETERS}

\subsection{The idea of Taguchi method}

Taguchi developed his methods in the 1950s and 1960s. Taguchi's approach to the product design process may be divided into three stages: system design, parameter design, and tolerance design [14-16]. System design is the conceptual design stage where the system configuration is developed. Parameter design, sometime called robust design, identifies factors that reduce the system sensitivity to noise, thereby enhancing the system's robustness. Tolerance design specifics the allowable deviations in the parameter values, loosening tolerances if possible and tightening tolerances if necessary. Taguchi's objective functions for robust design arise from quality measures using quadratic loss functions. In the extension of this definition to design optimisation. Taguchi suggested the signal-to-noise (SNR), $-10 \log _{10}(\mathrm{MSD})$, as a measure of the mean squared deviation (MSD) in the performance. The use of SNR in system analysis provides a quantitative value for response variation comparison.

The mathematical basis of the Taguchi method is mathematical methods of statistics. The Taguchi method allows determining the optimal condition of many parameters of the research object. This method is applied to solve the multi-objective optimization problem in mechanical engineering, civil engineering, and transportation engineering. In this paper, Taguchi's method is applied to optimize the parameters of DVA to reduce the vibration amplitude of primary system. By using the Taguchi method, we must note the following two important points. The first is that we need to determine the quality characteristics of the problem. The second option is that we need to select the orthogonal arrays. The Taguchi's methods begin with the definition of the word quality. Taguchi employs a revolutionary definition: "Quality is the loss imparted to society from the time a product is shipped" [20]. In this paper the quality characteristics are also called the signal-to-noise ratio (SNR). It is defined for a nominal-the-best procedure as $[15,16]$

$$
\eta=\mathrm{SNR}=-10 \log _{10}\left(H_{\text {actual }}-H_{\min }\right)^{2},
$$

where $H_{\text {actual }}$ is the target function in experiment $j$, and $H_{\min }$ is desired value of target function. Taguchi developed the orthogonal array method to study the systems in a convenient and rapid way, whose performance is affected by different factors when the considered system becomes more complicated with increasing number of influence factors.

\subsection{A procedure for optimal design}

This subsection aims to present numerical results that verify the procedure discussed above by using Taguchi's method [22]. Fig. 3 shows a beam with three dynamic translational vibration absorbers with damping placed at the points $\eta_{1}=3 L / 8, \quad \eta_{2}=4 L / 8, \eta_{3}=$ $5 \mathrm{~L} / 8$. The parameters of the beam are listed in Tab. 1.

Form Tab. 1 we can calculate $\omega_{1}=110.3005 \mathrm{rad} / \mathrm{s}, m_{b}=245 \mathrm{~kg}$. In which $m_{b}$ is the mass of the beam, and $\omega_{1}=110.3005 \mathrm{rad} / \mathrm{s}$ is the fundamental frequency of the beam. 


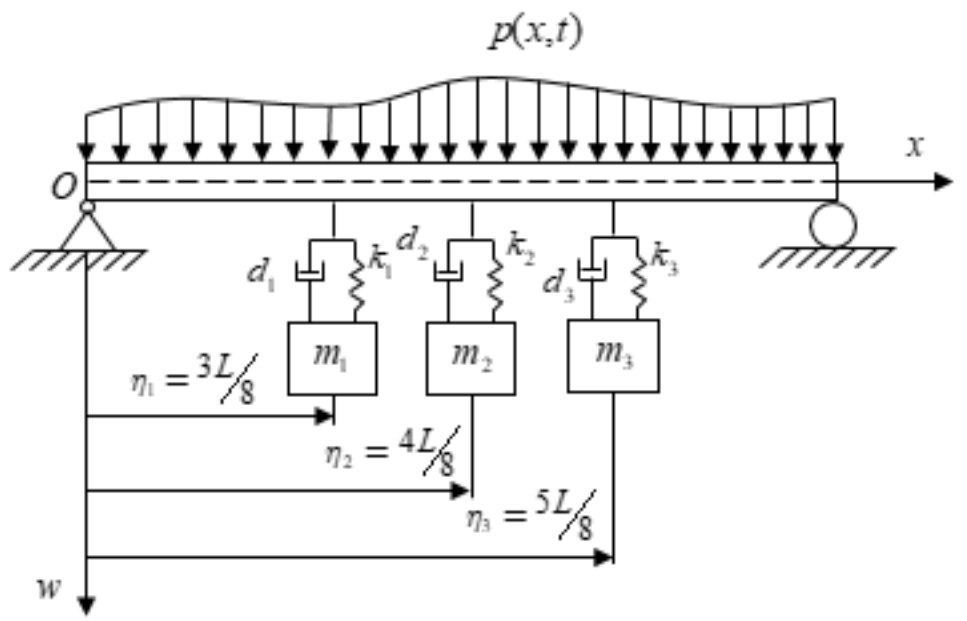

Fig. 3. Beam simply supported at both ends with three absorbers

Table 1. Parameters of beam

\begin{tabular}{lccc}
\hline \multicolumn{1}{c}{ Parameters } & Variable & Value & Unit \\
\hline Fexural rigidity & $E I$ & $3.06 \times 10^{7}$ & $\mathrm{Nm}^{2}$ \\
Length of beam & $L$ & 10 & $\mathrm{~m}$ \\
Mass per length unit & $\mu$ & 24.5 & $\mathrm{~kg} / \mathrm{m}$ \\
Damping coefficient & $c^{(e)}$ & 0.4 & $1 / \mathrm{s}$ \\
Internal friction coefficient & $c^{(i)}$ & 0.0005 & $\mathrm{~s} / \mathrm{m}$ \\
Distributed force & $p=p_{0} \sin \Omega t$ & $p_{0}=100$ & $\mathrm{~N} / \mathrm{m}$ \\
Coordinate of $j$-absorber & $\eta_{1}, \eta_{2}, \eta_{3}$ & $3 L / 8,4 L / 8,5 L / 8$ & $\mathrm{~m}$ \\
\hline
\end{tabular}

Step 1: Selection of control factors and target function

In the design experience, the engineer normally selects that the mass of DVAs is equal to $1 \%$ of the mass of beam. Accordingly, the masses of the DVAs can be chosen as follows

$$
m_{1}=\frac{0.1}{100} m_{b}=0.245 \mathrm{~kg}, \quad m_{2}=\frac{0.8}{100} m_{b}=1.960 \mathrm{~kg}, m_{3}=\frac{0.1}{100} m_{b}=0.245 \mathrm{~kg}
$$

The control factors are chosen as follows

$$
\mathbf{x}=\left[\begin{array}{llllll}
x_{1} & x_{2} & x_{3} & x_{4} & x_{5} & x_{6}
\end{array}\right]^{T}=\left[\begin{array}{llllll}
d_{1} & k_{1} & d_{2} & k_{2} & d_{3} & k_{3}
\end{array}\right]^{T} .
$$

The target function $H$ is chosen according to the formula (64)

$$
H=\left|H_{s}\right|=\frac{\left|\widehat{h}_{s}\right|}{\sqrt{a^{2}+b^{2}}} .
$$

Three levels of each control factor are given in Tab. 2 . 
Table 2. Control factors and levels of each control factor

\begin{tabular}{ccccccc}
\hline \multirow{2}{*}{ Levels } & \multicolumn{7}{c}{ Control factors } \\
\cline { 2 - 7 } & $d_{1}[\mathrm{Ns} / \mathrm{m}]$ & $k_{1}[\mathrm{~N} / \mathrm{m}]$ & $d_{2}[\mathrm{Ns} / \mathrm{m}]$ & $k_{2}[\mathrm{~N} / \mathrm{m}]$ & $d_{3}[\mathrm{Ns} / \mathrm{m}]$ & $k_{3}[\mathrm{~N} / \mathrm{m}]$ \\
\hline 1 & 0.005 & $1.0 \times 10^{4}$ & 0.1 & $2.0 \times 10^{4}$ & 0.004 & $0.5 \times 10^{4}$ \\
2 & 0.010 & $1.5 \times 10^{4}$ & 0.2 & $2.5 \times 10^{4}$ & 0.006 & $1.0 \times 10^{4}$ \\
3 & 0.015 & $2.0 \times 10^{4}$ & 0.3 & $3.0 \times 10^{4}$ & 0.008 & $1.5 \times 10^{4}$ \\
\hline
\end{tabular}

Step 2: Selection of orthogonal array and calculation of signal-to-noise ratio (SNR)

Three levels of each control factor are applied, necessitating the use of an L18 orthogonal array (Tab. 3) $[15,16]$. Coding stage 1, stage 2 , stage 3 of the control parameters by the symbols 1, 2,3. By performing the experiments and then calculating the corresponding response results, we have the values of the target function $H$ as shown in the Tab. 3, in which a target value of $H_{\min }=0$ is selected.

Table 3. Experimental design using L18 orthogonal array

\begin{tabular}{|c|c|c|c|c|c|c|c|c|}
\hline \multirow{2}{*}{ Trial } & \multicolumn{6}{|c|}{ Control factors } & \multicolumn{2}{|c|}{ Results } \\
\hline & $d_{1}$ & $k_{1}$ & $d_{2}$ & $k_{2}$ & $d_{3}$ & $k_{3}$ & $\mathrm{H}$ & SNR \\
\hline 1 & 1 & 1 & 1 & 1 & 1 & 1 & 0.0044340966 & 47.0638969423 \\
\hline 2 & 1 & 2 & 2 & 2 & 2 & 2 & 0.0012036545 & 58.3899629081 \\
\hline 3 & 1 & 3 & 3 & 3 & 3 & 3 & 0.0042376395 & 47.4575198849 \\
\hline 4 & 2 & 1 & 1 & 2 & 2 & 3 & 0.0012017250 & 58.4038977293 \\
\hline 5 & 2 & 2 & 2 & 3 & 3 & 1 & 0.0041602303 & 47.6176524925 \\
\hline 6 & 2 & 3 & 3 & 1 & 1 & 2 & 0.0043690768 & 47.1922063525 \\
\hline 7 & 3 & 1 & 2 & 1 & 3 & 2 & 0.0043767090 & 47.1770466279 \\
\hline 8 & 3 & 2 & 3 & 2 & 1 & 3 & 0.0012067156 & 58.3679013846 \\
\hline 9 & 3 & 3 & 1 & 3 & 2 & 1 & 0.0041617143 & 47.6145548175 \\
\hline 10 & 1 & 1 & 3 & 3 & 2 & 2 & 0.0042128399 & 47.5085009203 \\
\hline 11 & 1 & 2 & 1 & 1 & 3 & 3 & 0.0043474735 & 47.2352611858 \\
\hline 12 & 1 & 3 & 2 & 2 & 1 & 1 & 0.0011980787 & 58.4302931033 \\
\hline 13 & 2 & 1 & 2 & 3 & 1 & 3 & 0.0042203721 & 47.4929851721 \\
\hline 14 & 2 & 2 & 3 & 1 & 2 & 1 & 0.0044391926 & 47.0539202335 \\
\hline 15 & 2 & 3 & 1 & 2 & 3 & 2 & 0.0012021408 & 58.4008931815 \\
\hline 16 & 3 & 1 & 3 & 2 & 3 & 1 & 0.0011986789 & 58.4259426044 \\
\hline 17 & 3 & 2 & 1 & 3 & 1 & 2 & 0.0042176774 & 47.4985327364 \\
\hline 18 & 3 & 3 & 2 & 1 & 2 & 3 & 0.0043506134 & 47.2289902086 \\
\hline
\end{tabular}

The experimental results are then analyzed by means of the mean square deviation of the target function for each control parameter, namely the calculation of the SNR of 
the control factors according to the formula

$$
\eta_{j}=(\mathrm{SNR})_{j}=-10 \log \left(H_{j}-H_{o p t}\right)^{2}, j=1, \ldots, 18,
$$

where $H_{j}$ is the target function in experiment $j$, and $H_{o p t}$ is desired value of target function.

Step 3: Analysis of signal-to-noise ratio (SNR)

From Tab. 3 we can calculate the mean value of the SNR of the control parameter of $d_{1}=x_{1}$ corresponds to the levels $1,2,3$

$\operatorname{SNR}\left(x_{1}^{1}\right)=(\operatorname{SNR}(1)+\operatorname{SNR}(2)+\operatorname{SNR}(3)+\operatorname{SNR}(10)+\operatorname{SNR}(11)+\operatorname{SNR}(12)) / 6=51.0142391574$,
$\operatorname{SNR}\left(x_{1}^{2}\right)=(\operatorname{SNR}(4)+\operatorname{SNR}(5)+\operatorname{SNR}(6)+\operatorname{SNR}(13)+\operatorname{SNR}(14)+\operatorname{SNR}(15)) / 6=51.0269258602$,
$\operatorname{SNR}\left(x_{1}^{3}\right)=(\operatorname{SNR}(7)+\operatorname{SNR}(8)+\operatorname{SNR}(9)+\operatorname{SNR}(16)+\operatorname{SNR}(17)+\operatorname{SNR}(18)) / 6=51.0521613966$.

In which $\operatorname{SNR}\left(x_{1}^{1}\right), \operatorname{SNR}\left(x_{1}^{2}\right), \operatorname{SNR}\left(x_{1}^{3}\right)$ are the mean square deviation of the control parameter $d_{1}$ at the levels $1,2,3$, respectively. Similarly we calculate the mean square deviation of the SNR for the levels 1,2, 3 of the control parameter $k_{1}=x_{2}, d_{2}=x_{3}, k_{2}=$ $x_{4}, d_{3}=x_{5}, k_{3}=x_{6}$

$\operatorname{SNR}\left(x_{2}^{1}\right)=(\operatorname{SNR}(1)+\operatorname{SNR}(4)+\operatorname{SNR}(7)+\operatorname{SNR}(10)+\operatorname{SNR}(13)+\operatorname{SNR}(16)) / 6=51.0120449994$, $\operatorname{SNR}\left(x_{2}^{2}\right)=(\operatorname{SNR}(2)+\operatorname{SNR}(5)+\operatorname{SNR}(8)+\operatorname{SNR}(11)+\operatorname{SNR}(14)+\operatorname{SNR}(17)) / 6=51.0272051568$, $\operatorname{SNR}\left(x_{2}^{3}\right)=(\operatorname{SNR}(3)+\operatorname{SNR}(6)+\operatorname{SNR}(9)+\operatorname{SNR}(12)+\operatorname{SNR}(15)+\operatorname{SNR}(18)) / 6=51.0540762581$, $\operatorname{SNR}\left(x_{3}^{1}\right)=(\operatorname{SNR}(1)+\operatorname{SNR}(4)+\operatorname{SNR}(9)+\operatorname{SNR}(11)+\operatorname{SNR}(15)+\operatorname{SNR}(17)) / 6=51.0361727655$, $\mathrm{SNR}\left(x_{3}^{2}\right)=(\mathrm{SNR}(2)+\mathrm{SNR}(5)+\mathrm{SNR}(7)+\mathrm{SNR}(12)+\mathrm{SNR}(13)+\mathrm{SNR}(18)) / 6=51.0561550854$, $\operatorname{SNR}\left(x_{3}^{3}\right)=(\operatorname{SNR}(3)+\operatorname{SNR}(6)+\operatorname{SNR}(8)+\operatorname{SNR}(10)+\operatorname{SNR}(14)+\operatorname{SNR}(16)) / 6=51.0009985634$, $\operatorname{SNR}\left(x_{4}^{1}\right)=(\operatorname{SNR}(1)+\operatorname{SNR}(6)+\operatorname{SNR}(7)+\operatorname{SNR}(11)+\operatorname{SNR}(14)+\operatorname{SNR}(18)) / 6=47.1585535918$, $\operatorname{SNR}\left(x_{4}^{2}\right)=(\operatorname{SNR}(2)+\operatorname{SNR}(4)+\operatorname{SNR}(8)+\operatorname{SNR}(12)+\operatorname{SNR}(15)+\operatorname{SNR}(16)) / 6=58.4031484852$, $\operatorname{SNR}\left(x_{4}^{3}\right)=(\operatorname{SNR}(3)+\operatorname{SNR}(5)+\operatorname{SNR}(9)+\operatorname{SNR}(10)+\operatorname{SNR}(13)+\operatorname{SNR}(17)) / 6=47.5316243373$, $\operatorname{SNR}\left(x_{5}^{1}\right)=(\operatorname{SNR}(1)+\operatorname{SNR}(6)+\operatorname{SNR}(8)+\operatorname{SNR}(12)+\operatorname{SNR}(13)+\operatorname{SNR}(17)) / 6=51.0076359485$, $\operatorname{SNR}\left(x_{5}^{2}\right)=(\operatorname{SNR}(2)+\operatorname{SNR}(4)+\operatorname{SNR}(9)+\operatorname{SNR}(10)+\operatorname{SNR}(14)+\operatorname{SNR}(18)) / 6=51.0333044696$, $\operatorname{SNR}\left(x_{5}^{3}\right)=(\operatorname{SNR}(3)+\operatorname{SNR}(5)+\operatorname{SNR}(7)+\operatorname{SNR}(11)+\operatorname{SNR}(15)+\operatorname{SNR}(16)) / 6=51.0523859962$, $\operatorname{SNR}\left(x_{6}^{1}\right)=(\operatorname{SNR}(1)+\operatorname{SNR}(5)+\operatorname{SNR}(9)+\operatorname{SNR}(12)+\operatorname{SNR}(14)+\operatorname{SNR}(16)) / 6=51.0343766989$, $\operatorname{SNR}\left(x_{6}^{2}\right)=(\operatorname{SNR}(2)+\operatorname{SNR}(6)+\operatorname{SNR}(7)+\operatorname{SNR}(10)+\operatorname{SNR}(15)+\operatorname{SNR}(17)) / 6=51.0278571211$, $\operatorname{SNR}\left(x_{6}^{3}\right)=(\operatorname{SNR}(3)+\operatorname{SNR}(4)+\operatorname{SNR}(8)+\operatorname{SNR}(11)+\operatorname{SNR}(13)+\operatorname{SNR}(18)) / 6=51.0310925942$.

In which $\operatorname{SNR}\left(x_{1}^{1}\right), \operatorname{SNR}\left(x_{1}^{2}\right), \operatorname{SNR}\left(x_{1}^{3}\right)$ are the mean square deviation of the control parameter $d_{1}$ at the levels $1,2,3$, respectively. Similarly we calculate the mean square deviation of the SNR for the levels 1, 2, 3 of the control parameter $k_{1}=x_{2}, d_{2}=x_{3}, k_{2}=$ $x_{4}, d_{3}=x_{5}, k_{3}=x_{6}$. Then SNR Ratio can be plotted to use for optimization of seat displacement as shown in Fig. 4. 

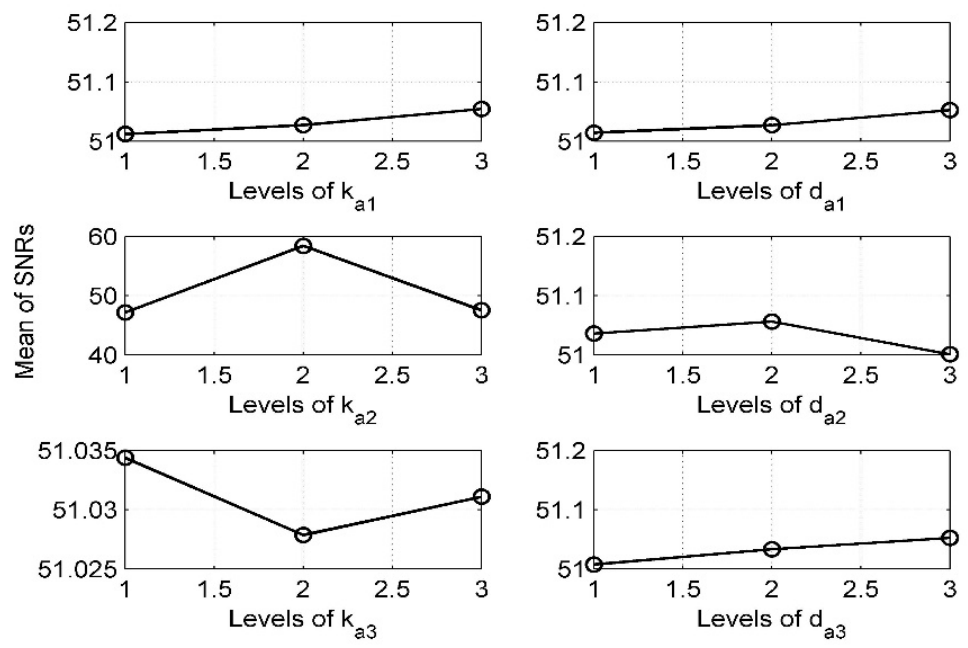

Fig. 4. SNR Ratio plot for optimization of seat displacement of control parameters $d_{1}, k_{1}, d_{2}, k_{2}, d_{3}, k_{3}$

From Fig. 4 the optimal signal-to-noise ratio of the control parameters can be derived as follows

$$
\begin{aligned}
& (\mathrm{SNR}) x_{1}=51.0521613966,(\mathrm{SNR}) x_{2}=51.0540762581, \\
& (\mathrm{SNR}) x_{3}=51.0561550854,(\mathrm{SNR}) x_{4}=58.4031484852, \\
& (\mathrm{SNR}) x_{5}=51.0523859962,(\mathrm{SNR}) x_{6}=51.0343766989 .
\end{aligned}
$$

Step 4: Selection of new levels for control factors

From Eq. (40) it can be seen that the optimal SNR of the control parameters is different. This makes it easy to perform iterative calculation. Firstly new levels for control parameters are selected. Based on the level distribution diagram of the parameter as shown in Fig. 4, we choose the new levels of control parameters as follows. The optimal parameters are levels with the largest value of the parameters, namely, $d_{1}$ level $3, k_{1}$ level $3, d_{2}$ level $2, k_{2}$ level $2, d_{3}$ level $3, k_{3}$ level 1 . Therefore, we have the values of the new levels as follows:

If level 1 is optimal then the next levels are

$$
\begin{gathered}
\text { New level 1 level 1 } \\
\left\{\begin{array}{l}
\text { level2_new }=\text { level1_old } \\
\text { level1_new }=\text { level1_old }-\frac{\text { level2_old }- \text { level1_old }}{2} \\
\text { level3_new }=\text { level1_old }+\frac{\text { level2_old }- \text { level1_old }}{2}
\end{array}\right.
\end{gathered}
$$

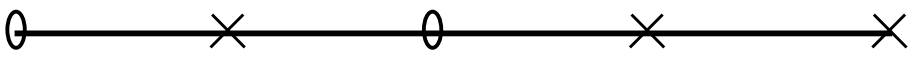

If level 2 is optimal then the next levels are 


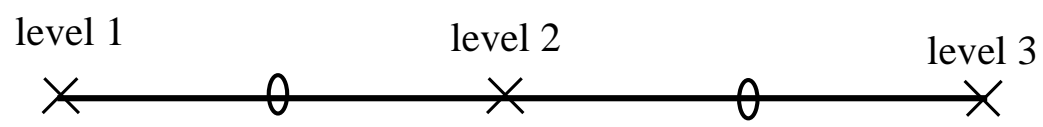

New level 1 New level 2 New level 3

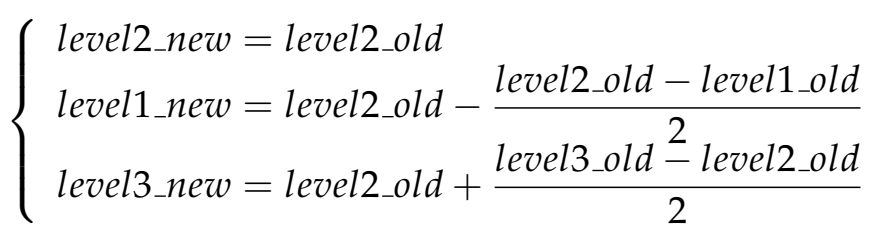

If level 3 is optimal then the next levels are

$$
\begin{aligned}
& \text { level } 1 \quad \text { level } 2 \quad \text { level } 3 \quad \text { New level } 3 \\
& \text { New level } 1 \quad \text { New level } 2 \\
& \left\{\begin{array}{l}
\text { level2_new }=\text { level3_old } \\
\text { level1_new }=\text { level3_old }-\frac{\text { level3_old }- \text { level2_old }}{2} \\
\text { level3_new }=\text { level3_old }+\frac{\text { level3_old }- \text { level2_old }}{2}
\end{array}\right.
\end{aligned}
$$

According to the above rule, we have the new levels of control parameters as shown in Tab. 4.

Table 4. Control factors and new levels of control factors

\begin{tabular}{ccccccc}
\hline \multirow{2}{*}{ Levels } & \multicolumn{7}{c}{ Control factors } \\
\cline { 2 - 7 } & $d_{1}[\mathrm{Ns} / \mathrm{m}]$ & $k_{1}[\mathrm{~N} / \mathrm{m}]$ & $d_{2}[\mathrm{Ns} / \mathrm{m}]$ & $k_{2}[\mathrm{~N} / \mathrm{m}]$ & $d_{3}[\mathrm{Ns} / \mathrm{m}]$ & $k_{3}[\mathrm{~N} / \mathrm{m}]$ \\
\hline 1 & 0.0125 & $1.75 \times 10^{4}$ & 0.1500 & $2.25 \times 10^{4}$ & 0.0700 & $0.25 \times 10^{4}$ \\
2 & 0.0150 & $2.00 \times 10^{4}$ & 0.2000 & $2.50 \times 10^{4}$ & 0.0800 & $0.50 \times 10^{4}$ \\
3 & 0.0175 & $2.25 \times 10^{4}$ & 0.2500 & $2.75 \times 10^{4}$ & 0.0900 & $0.75 \times 10^{4}$ \\
\hline
\end{tabular}

Then the analysis of signal-to-noise ratio (SNR) is performed as the step 2.

Step 5: Check the convergence condition of the signal-to-noise ratio and determine the optimal parameters of the DVA

After 24 iterations, we obtain the optimal noise values of the control parameters. The calculation results are recorded in Tab. 5 .

If the optimal signal-to-noise ratio of the control parameters is equal (or approximately equal) we move on to step 5. If otherwise we return to step 2. According to 
Table 5. Noise values of the control parameter $(\mathrm{SNR})_{i}$ of the control parameters

\begin{tabular}{ccccccc}
\hline \multirow{2}{*}{ Trial } & \multicolumn{5}{c}{ Optimal noise values $(\mathrm{SNR}){ }_{i}$} \\
\cline { 2 - 7 } & $(\mathrm{SNR}) x_{1}$ & $(\mathrm{SNR}) x_{2}$ & $(\mathrm{SNR}) x_{3}$ & $(\mathrm{SNR}) x_{4}$ & $(\mathrm{SNR}) x_{5}$ & $(\mathrm{SNR}) x_{6}$ \\
\hline 1 & 51.052161 & 51.054076 & 51.056155 & 58.403148 & 51.052386 & 51.034377 \\
2 & 54.879695 & 54.866990 & 54.859337 & 58.311646 & 54.865234 & 54.870096 \\
3 & 63.488241 & 63.473320 & 63.495179 & 78.961093 & 63.496090 & 63.533600 \\
$\ldots$ & $\ldots$ & $\ldots$ & $\ldots$ & $\ldots$ & $\ldots$ & $\ldots$ \\
23 & 91.588713 & 91.588713 & 91.588714 & 91.588713 & 91.588713 & 91.588713 \\
24 & 91.588714 & 91.588714 & 91.588714 & 91.588714 & 91.588714 & 91.588714 \\
\hline
\end{tabular}

the above analysis, we obtain the optimal values of the parameter of absorber after 24 iterations as

$$
\begin{aligned}
& m_{1}=0.2450(\mathrm{~kg}), d_{1}=0.0145(\mathrm{Ns} / \mathrm{m}), k_{1}=1.9652 \times 10^{4}(\mathrm{~N} / \mathrm{m}), \\
& m_{2}=1.960(\mathrm{~kg}), d_{2}=0.2125(\mathrm{Ns} / \mathrm{m}), k_{2}=2.3846 \times 10^{4}(\mathrm{~N} / \mathrm{m}), \\
& m_{3}=0.2450(\mathrm{~kg}), d_{3}=0.0779(\mathrm{Ns} / \mathrm{m}), k_{3}=0.4470 \times 10^{4}(\mathrm{~N} / \mathrm{m}) .
\end{aligned}
$$

Step 6: Determine the vibration of the primary system and of the DVA

Knowing the parameters of the DVAs, using Eq. (41) we can easily calculate the vibration of the beam with DVAs and without DVAs. Fig. 5 shows the response of the beam at the $x=L / 2$ with DVAs and without DVAs. It can be clearly seen that the vibration amplitude of the beam at $x=L / 2$ without DVAs is $7.267(\mathrm{~mm})$, and reduces to 0.03068 $(\mathrm{mm})$ with 3 DVAs at $3 L / 8,4 L / 8,5 \mathrm{~L} / 4$ and the reducing rate is $99.57 \%$ at the excitation frequency equal to the first natural frequency of the beam.

\subsection{Problem formulation for determining optimal parameters of DVAs in frequency domain}

When a primary system is damped, the "fixed-points" feature no longer exists. However, as shown in the work of Pannestri [23], when a DVA with a small mass ratio is attached to lightly or moderately damped primary systems, the normalized amplitude curves roughly join at two points. When the damping ratio of the primary system approaches zero, these two points converge to the "fixed-points". Therefore, it is justified to assume that the "fixed-point" theory also approximately holds even for the case when a DVA is attached to a lightly or moderately damped primary system. Based on this assumption, it is reasonable to assume that $H(\Omega)$ has two distinct resonance points, Liu and Coppola [24]. These are denoted by $\mathrm{A}$ and $\mathrm{B}$ with frequencies $\Omega_{A}$ and $\Omega_{B}\left(\Omega_{A}<\Omega_{B}\right)$, respectively. This leads to the equations

$$
H\left(\Omega_{A}\right)=\max |H(\Omega)| \text { and } H\left(\Omega_{B}\right)=\max |H(\Omega)| \text {. }
$$

It is well recognized that each fixed point very close to the corresponding resonance point, and that the trade off relation between $H\left(\Omega_{A}\right)=\max |H(\Omega)|$ and $H\left(\Omega_{B}\right)=$ $\max |H(\Omega)|$ can be postulated. On this assumption, it is guaranteed that the optimum 


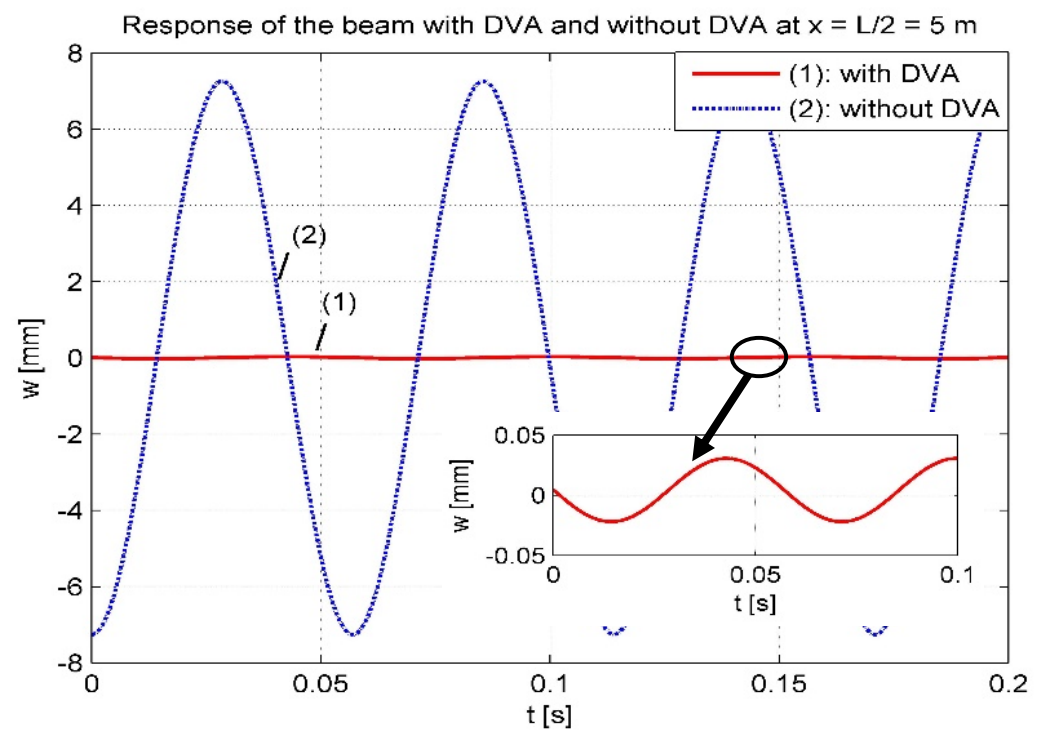

Fig. 5. The response of the beam at $x=L / 2=5 \mathrm{~m}$ without and with 3 DVAs at $3 L / 8,4 L / 8,5 L / 8$

design is derived using equivalent resonance magnification factors

$$
\max |H(\Omega)|=\left|H\left(\Omega_{A}\right)\right|=\left|H\left(\Omega_{B}\right)\right| .
$$

The problem can also be formulated as the one that minimizes the following two functions

$$
\begin{aligned}
& f_{1}=\frac{1}{2}\left|H\left(\Omega_{A}\right)-H\left(\Omega_{B}\right)\right|, \\
& f_{2}=\frac{1}{2}\left|H\left(\Omega_{S}\right)+H\left(\Omega_{B}\right)\right| .
\end{aligned}
$$

A target function can be defined as

$$
f=\varepsilon_{1} f_{1}+\varepsilon_{2} f_{2} \rightarrow \min ,
$$

where $\varepsilon_{1}$ and $\varepsilon_{2}$ are weighting factors used to impose different emphasis on each of the target functions.

Using the optimal parameters of the DVAs in Eq. (41), we can plot the amplitudefrequency curve as shown in Fig. 6. Figs. 5 and 6 show the response of the beam at $x=L / 2=5 \mathrm{~m}$ without DVAs is $7.267 \mathrm{~mm}$ and reduces to $1.721 \mathrm{~mm}$ with three DVAs in in a narrow band of the resonance frequency. The reducing rate is $76.32 \%$.It can be seen form Fig. 6 that three resonance nodes at the frequencies $\Omega_{A}, \Omega_{s}, \Omega_{B}$. From there we have $\Omega_{A}=0.94 \Omega_{s}, \Omega_{B}=1.1053 \Omega_{s}$.

According to Eq. (41), we define the new optimal parameters of DVAs by Taguchi's method. Thus the amplitude-frequency curves with the weighting factors $\varepsilon_{1}=0.8, \varepsilon_{2}=$ 0.3 with will be drawn as in Fig. 7. The amplitude-frequency curve is also called the compliance curve. From Fig. 7, it is found that the maximum vale of the vibration amplitude corresponds to the case of the beam having installed 3 DVAs is $1.67 \mathrm{~mm}$. The vibration 
amplitude of the beam without DVAs at $x=L / 2=5 \mathrm{~m}$ is $7.267 \mathrm{~mm}$. The vibration amplitude of the beam decreased by $77.02 \%$.

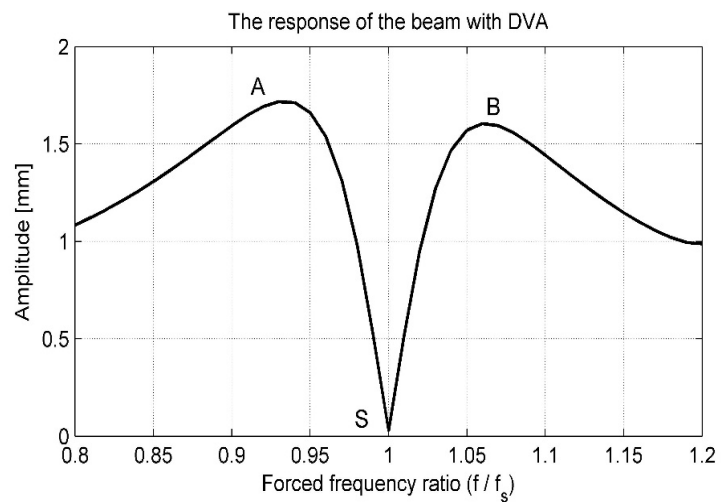

Fig. 6. Amplitude-Frequency curve in a narrow band of the resonance frequency

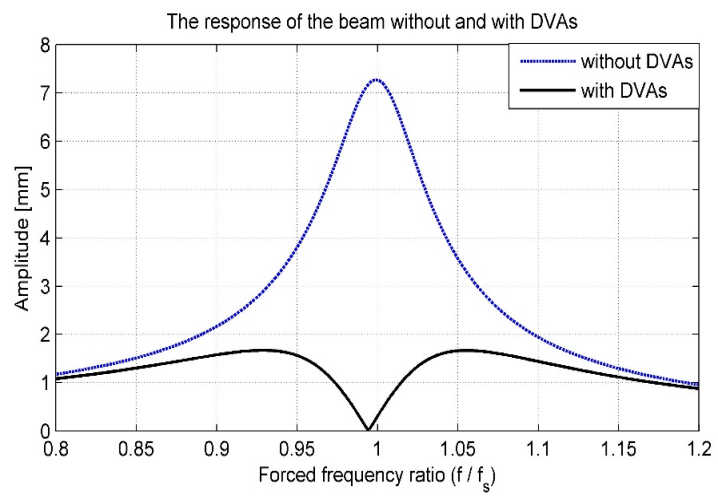

Fig. 7. The compliance curve in in a narrow band of the resonance frequency

\section{CONCLUSIONS}

When a damped primary system is excited by a harmonic force, its vibration can be suppressed by attaching a DVA. The DVA has the effect of reducing vibrations in the resonance region, and has almost ineffective far out of the resonance region. In this paper, the performance of the optimal design of parameters of a number of DVAs installed in an Euler-Bernoulli beam was investigated from the viewpoint of suppressing vibration amplitude of the beam. Based on the obtained results, the following concluding remarks can be reached.

- A general method for derivation of transverse vibration equations of beam with dynamic vibration absorbers is presented.

- A procedure based on the Taguchi's method for designing the optimal parameters for the DVAs attached to the beam is proposed. The use of the Taguchi's method to design the optimal parameters of the DVAs installed in damped primary system is relatively simple and convenient.

- The Taguchi's method has the good effect of reducing vibration in a narrow band of the resonance frequency (the ratio is approximately $80 \%$ ).

\section{ACKNOWLEDGEMENTS}

This paper was completed with the financial support of the Vietnam National Foundation for Science and Technology Development (NAFOSTED) under grant number 107.04-2017.04

\section{REFERENCES}

[1] D. J. Inman. Engineering vibration. Prentice-Hall, Inc., New Jersey, (2001). 
[2] P. Hagedorn and A. DasGupta. Vibrations and waves in continuous mechanical systems. John Wiley \& Sons, (2007).

[3] D. Hartog. Mechanical vibrations. McGraw-Hill, New York, (1956).

[4] B. G. Korenev and L. M. Reznikov. Dynamic vibration absorbers: theory and technical applications. John Wiley \& Sons, (1993).

[5] R. G. Jacquot. Optimal dynamic vibration absorbers for general beam systems. Journal of Sound and Vibration, 60, (4), (1978), pp. 535-542. https://doi.org/10.1016/s0022460x(78)80090-x.

[6] H. N. Özgüven and B. Candir. Suppressing the first and second resonances of beams by dynamic vibration absorbers. Journal of Sound and Vibration, 111, (3), (1986), pp. 377-390. https://doi.org/10.1016/s0022-460x(86)81399-2.

[7] Y. H. Lin and C. H. Cho. Vibration suppression of beam structures traversed by multiple moving loads using a damped absorber. Journal of Marine Science and Technology, 1, (1), (1993), pp. 39-48.

[8] M. Ouled Chtiba, S. Choura, S. El-Borgi, and A. H. Nayfeh. Confinement of vibrations in flexible structures using supplementary absorbers: dynamic optimization. Journal of Vibration and Control, 16, (3), (2010), pp. 357-376. https://doi.org/10.1177/1077546309103423.

[9] N. Carpineto, W. Lacarbonara, and F. Vestroni. Mitigation of pedestrian-induced vibrations in suspension footbridges via multiple tuned mass dampers. Journal of Vibration and Control, 16, (5), (2010), pp. 749-776. https://doi.org/10.1177/1077546309350188.

[10] B. Noori and A. Farshidianfar. Optimum design of dynamic vibration absorbers for a beam, based on $\mathrm{H}_{\infty}$ and $\mathrm{H}_{2}$ optimization. Archive of Applied Mechanics, 83, (12), (2013), pp. 17731787. https://doi.org/10.1007/s00419-013-0777-y.

[11] F. S. Samani, F. Pellicano, and A. Masoumi. Performances of dynamic vibration absorbers for beams subjected to moving loads. Nonlinear Dynamics, 73, (1-2), (2013), pp. 1065-1079. https://doi.org/10.1007/s11071-013-0853-4.

[12] S. S. Patil and P. J. Awasare. Vibration reduction at desired locations on a beam by creating nodes using tunable vibration neutralizers. Journal of Vibration and Control, 22, (1), (2016), pp. 205-223. https://doi.org/10.1177/1077546314528964.

[13] W. Łatas. Optimal positions of tunable translational and rotational dynamic absorbers in global vibration control in beams. Journal of Theoretical and Applied Mechanics, 53, (2), (2015), pp. 467-476. https://doi.org/10.15632/jtam-pl.53.2.467.

[14] W. Łatas. Application of the continuous dynamic absorbers in local and global vibration reduction problems in beams. Vibrations in Physical Systems, 27, (2016), pp. 245-254.

[15] G. Taguchi, S. Chowdhury, and Y. Wu. Taguchi's quality engineering handbook. John Wiley \& Sons, New Jersey, (2005).

[16] R. K. Roy. A primer on the Taguchi method. Society of Manufacturing Engineers, Michigan, (1990).

[17] B. Klein. Versuchsplanung-DoE: Einführung in die Taguchi/Shainin-Methodik. Oldenbourg Verlag, München, (2011).

[18] R. A. Zambanini. The application of Taguchi's method of parameter design to the design of mechanical systems. Master's thesis, Lehigh University, (1992).

[19] C. Zang, M. I. Friswell, and J. E. Mottershead. A review of robust optimal design and its application in dynamics. Computers $\mathcal{E}$ Structures, 83, (4-5), (2005), pp. 315-326. https://doi.org/10.1016/j.compstruc.2004.10.007.

[20] K. Dehnad. Quality control, robust design, and the Taguchi method. Pacific Grove, California, (1989). 
[21] N. T. Hung and P. X. Son. Experimental design in mechanical engineering. Construction Publishing House, Hanoi, (2016). (in Vietnamese).

[22] N. V. Khang, V. D. Phuc, D. T. Duong, and N. T. V. Huong. A procedure for optimal design of a dynamic vibration absorber installed in the damped primary system based on Taguchi's method. Vietnam Journal of Science and Technology, (2018). (Accepted).

[23] E. Pennestri. An application of Chebyshev's min-max criterion to the optimal design of a damped dynamic vibration absorber. Journal of Sound and Vibration, 217, (4), (1998), pp. 757765. https://doi.org/10.1006/jsvi.1998.1805.

[24] K. Liu and G. Coppola. Optimal design of damped dynamic vibration absorber for damped primary systems. Transactions of the Canadian Society for Mechanical Engineering, 34, (1), (2010), pp. 119-135. https://doi.org/10.1139/tcsme-2010-0008. 